\title{
Effect of Addition of Dexmedetomidine as an Adjuvant to Levobupivacaine in Supraclavicular Brachial Plexus Block
}

\author{
Authors \\ Dr Aneesh K V $\mathbf{1}^{\mathbf{1}}$, Dr Rajani Gandhi \\ ${ }^{1,2}$ Assistant Professor, Government Medical College Trivandrum
}

\section{INTRODUCTION}

Brachial plexus nerve blocks have become important in clinical practice because of their role in post-operative pain relief $^{1}$, shortening of patient recovery time and avoiding risks and adverse effects of General anaesthesia ${ }^{2,3}$. Hence,peripheral nerve blockade is now a well-accepted concept for comprehensive anesthetic care. In 1928, Kulenkampff and Persky published their experiences with a thousand blocks without apparent major complications $^{3}$. The subclavian (Supraclavicular) perivascular technique was first developed by Alon Willie and Collins ${ }^{4}$. The advantage of Supra clavicular technique over other brachial plexus approaches are its rapid onset and complete predictable anesthesia. Levobupivacaine is a high potent ,long acting amide local anaesthetic, with less cardio toxic effects ${ }^{5}$. Levobupivacaine is a local anesthetic with long duration, having similar pharmacology to bupivacaine; however, it has a wider safety margin and was shown to possess less cardio toxicity in comparison with bupivacaine $^{6,7}$. So it has been one of the most commonly used local anesthetic for peripheral nerve blocks. Dexmedetomidine is a pharmacologically active dextro isomer of Medetomidine; a selective $\alpha 2$-adrenergic receptor agonist which provides analgesia and sedation ${ }^{8}$, it provides more hemodynamic stability intense analgesia with less respiratory depression and rapid recovery ${ }^{8,9,10}$. Numerous studies has shown that the use of local anesthetic agents with adjuvants such as clonidine $^{16}$ and tramadol for a brachial plexus block to improve the quality and duration of anesthesia, and these studies have shown that the adjuvants may prolong anesthesia and analgesia. Hence here is an attem pt to study the Effect of addition of Dexmedetomidine to Levobupivacaine in supraclavicular brachial plexus block alone for supraclavicular brachial plexus block in prolonging the duration of analgesia in patients undergoing orthopedic surgery of forearm or hand. In this study, we investigated the effects of adding dexmedeto-midine to Levobupivacaine in supraclavicular brachial plexus block. The primary outcome of our study was the duration of analgesia, and the secondary outcome were duration and onset of sensory and motor block and side effects.

\section{MATERIALS AND METHODS}

After getting ethical committee approval and informed consent, a prospective cohort study to compare the effectiveness of $0.5 \%$ levobupivacaine versus $0.5 \%$ levobupivacaine with dexmedetomidine was conducted in Government 
Medical College, Trivandrum, during a period of one year. After Research Methodology and Ethical Committee approval for the study, study subjects are selected from among those coming for orthopedic surgeries of forearm or hand during the period of study at Medical college hospital. Subjects are assessed in the pre-anesthetic assessment clinic and categorized in to ASA classes. 60 Patients belonging to ASA class 1,2 and satisfying the inclusion and exclusion criteria are considered for the study. No premedication was given. No additional sedative medication was given Brachial plexus block is given with nerve stimulator with 22 gauge, insulated, unipolar needle. Motor response was obtained with $0.5 \mathrm{~mA}$ and a volume of $30 \mathrm{ml}$ of solutions injected.Intra operative monitoring include ECG, pulse oximetry, Noninvasive blood pressure was determined at 1 st minute and then at 5, 10, 30, $60,120,180,240,360$ and $480 \mathrm{~min}$ after completion of injection.

> Group A received 0.5\% levobupivacaine $30 \mathrm{~mL}+1 \mathrm{ml}$ dexmedetomidine. Total 31 $\mathrm{mL}$ solution.

$>$ Group B received 0.5\% levobupivacaine $30 \mathrm{~mL}+1 \mathrm{ml} 0.9 \%$ saline. Total $31 \mathrm{~mL}$ solution.

The duration of analgesia, onset and duration of sensory block, onset and duration of motor block, heart rate, blood pressure and sedation were recorded at the first minute and at 5, 10, 30, $60,120,180,240,360$ and $480 \mathrm{~min}$ after completion of injection.

Sensory block was determined by the pin prick test. Patient was requested to compare the pinprick ( $26 \mathrm{G}$ needle) sensation on the test arm to that in contralateral arm as reference (100 points). Scale range (0-no sensation to 100 -full sensation). Sensory block onset is defined as reduction in sensibility to $30 \%$ or less.

Duration of sensory block is defined as the time interval between injection and complete recovery of sensation. Patients were asked to note the complete recovery of sensation.
VAS score for analgesia 0-3: Good analgesia, 36:Moderate analgesia, 6-10: Poor analgesia Sensory and Motor block will be assessed by Hollmen scale.

\section{HOLLMEN SCALE}

\section{Sensory block}

$1=$ normal sensation of pinprick, $2=$ pin prick felt as sharp pointed but weaker compared with same area in the other upper limb, 3=pin prick recognized as touch with blunt object, 4=no perception of pin prick

Motor block

$1=$ normal muscle function, $2=$ slight weakness in function, $3=$ very weak muscular action, $4=$ complete loss of muscle action

Sedation score ranges from 1 (alert) to 4(asleep, not arousable by verbal contact). Sedation score

1 = awake, 2 =drowsy, $3=$ asleep but arousable, $4=$ asleep but not arousable. The highest sedation score in first 2 hours after injection was taken as the sedation score of the patient for statistical purposes. Occurrence of hypotension (fall of mean blood pressure by $>30 \%$ of baseline) anytime during the monitored period was classified as presence of hypotension.

Bradycardia ( $<45 \mathrm{bpm})$ will also be monitored. Analgesic effect was measured by the time of injection to the first demand of analgesic.

Measure of outcome Duration of analgesia is taken as the time between onset of analgesia and the reappearance of pain $(6-10 \mathrm{cms}$ in VAS) or request for pain relief

Onset of complete sensory block was considered complete when scores were (grade 4 in Hollmen) that will be checked by pin prick method over C5 TO T1 dermatomes

Onset of complete motor block was considered complete at a score of grade 4 in Hollmen Duration of motor block is taken as the time between complete motor block and return of the normal muscle function.

Quality of analgesia Patients were asked to provide a verbal rating of the quality (excellent, good, fair, poor) at the end of surgery. 
The block was considered to have failed if complete sensory and motor block was not achieved after 30 minutes and the failed block was converted to GA and it was recorded.

The duration of analgesia, onset and duration of sensory block, onset and duration of motor block, heart rate and blood pressure were recorded at the first minute and at 5, 10, 30, 60,120, 180, 240, 360 and $480 \mathrm{~min}$ after completion of injection.

\section{Statistical Analysis}

Data were analyzed using computer software, Statistical Package for Social Sciences (SPSS) version 10. Data are expressed in its frequency and percentage as well as mean and standard deviation. Student's $t$ test was used to compare mean values between two groups to elucidate the associations and comparisons between different parameters, chi square $\left(\chi^{2}\right)$ test was used as nonparametric test. Mann Whitney $U$ test was employed as non parametric test to compare the scores. For all statistical evaluations, a two-tailed probability of value, $\mathrm{P}<0.05$ was considered significant.

\section{OBSERVATION AND RESULTS}

Figure 1: Graph showing mean heart rate (bpm) at various time intervals

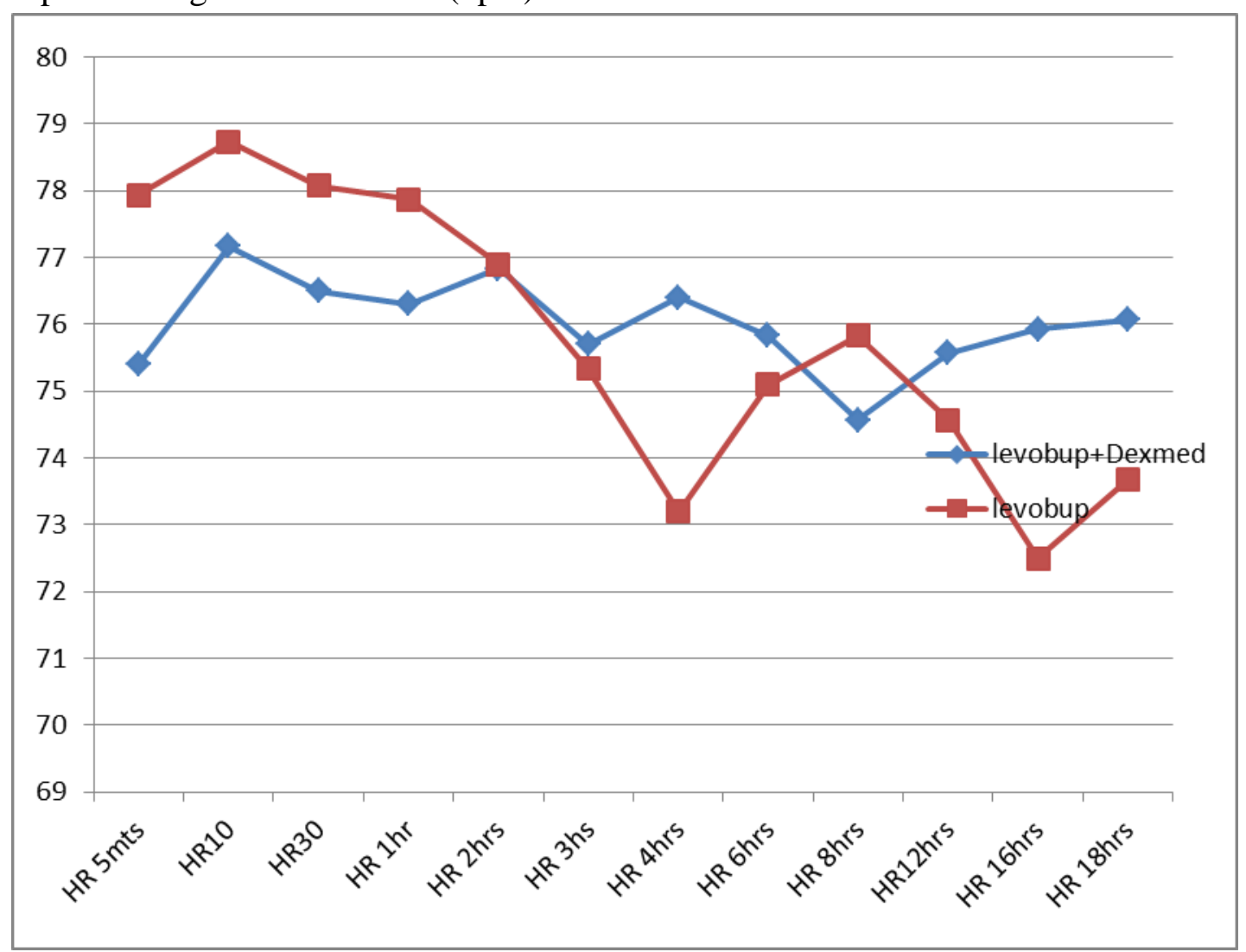

There is no statistically significant difference in heart rate between both the groups. 
Figure 2 Graph showing mean systolic blood pressure $(\mathrm{mmHg})$ at various time intervals

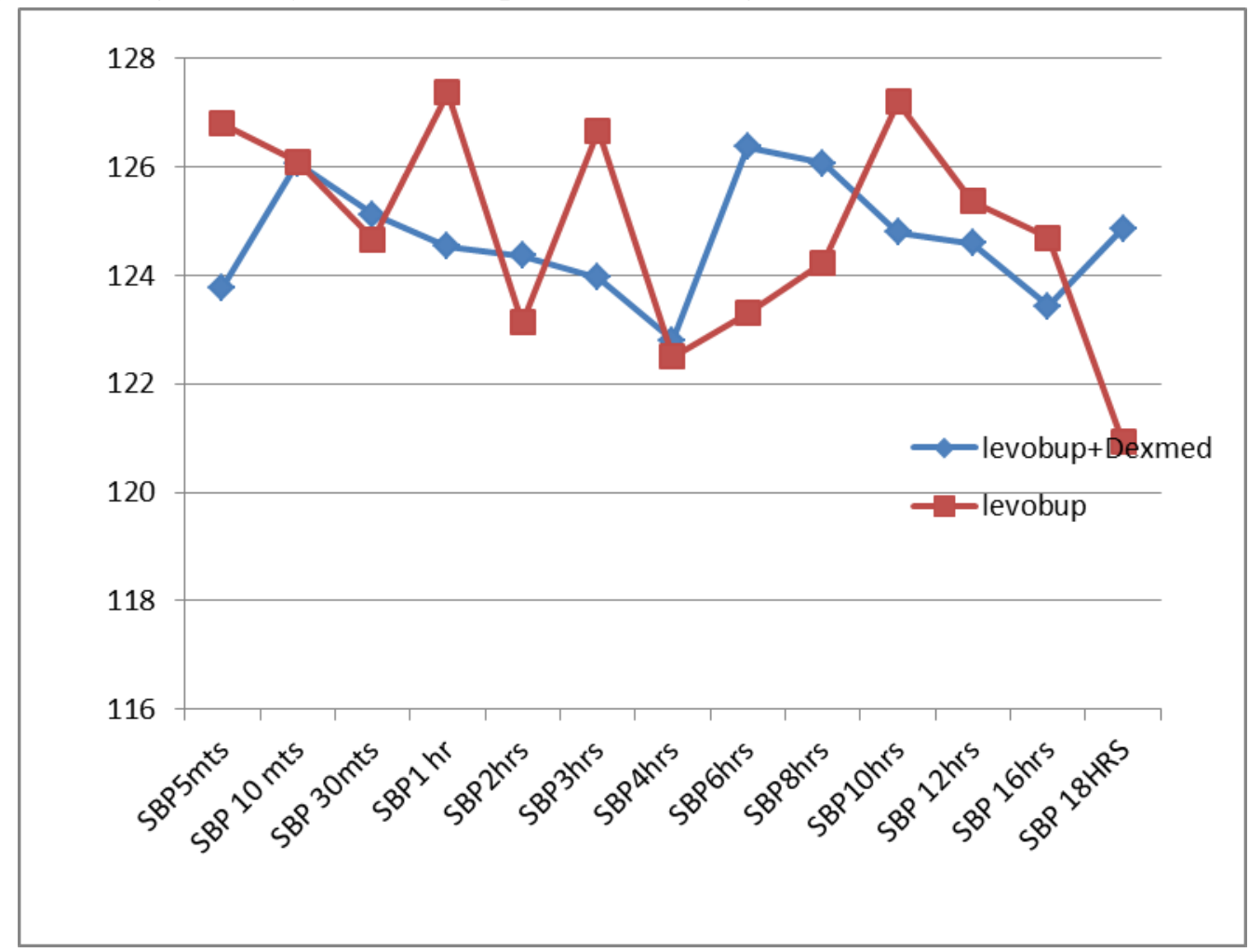

There is no statistically significant difference in systolic blood pressure between both the groups

Figure 3 Graph showing Diastolic blood pressure (DBP) at various time intervals

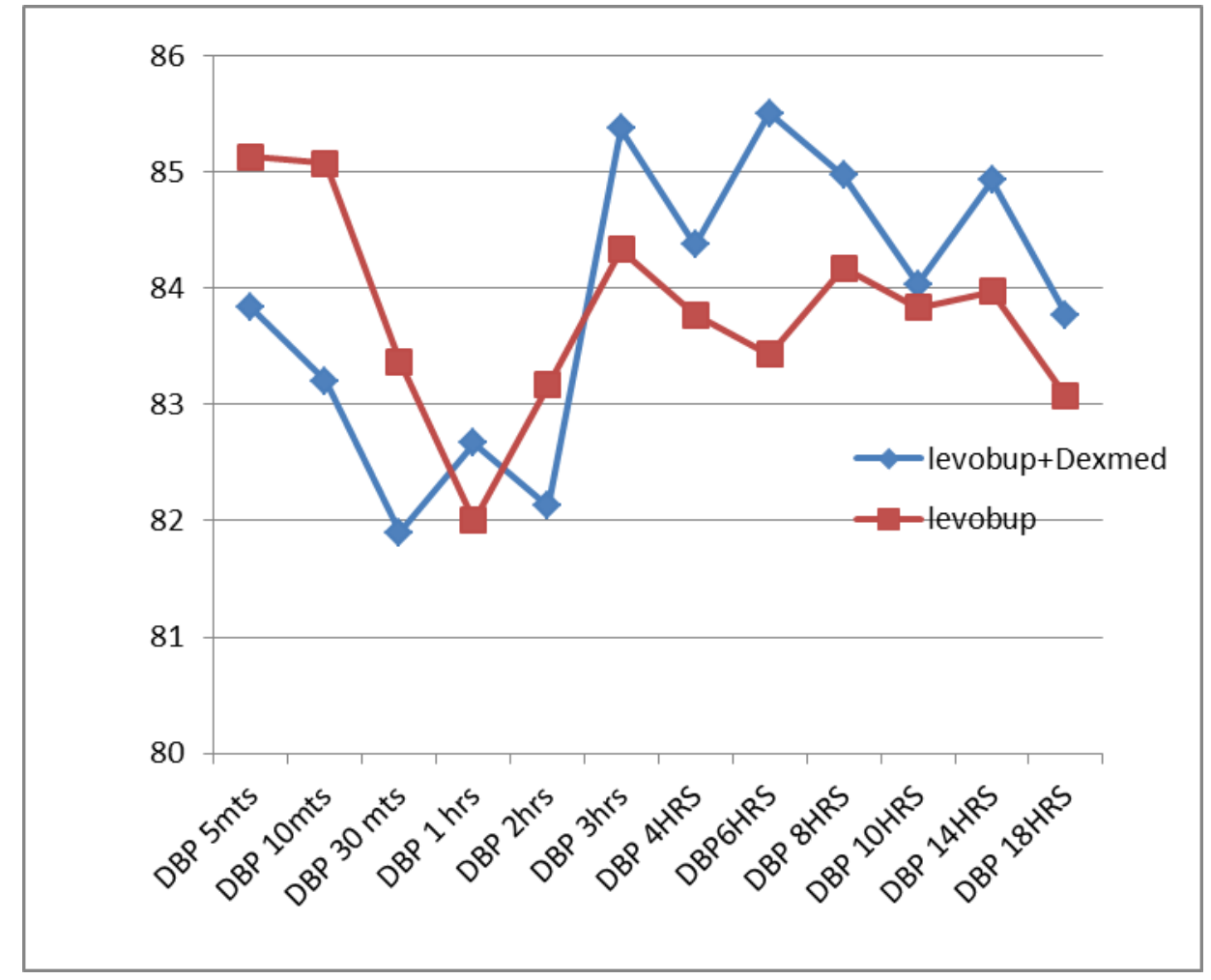

There is no statistically significant difference in diastolic blood pressure between both the groups. 
Mean onset time of motor block in Group A was 8.67 minutes with SD of 1.68 and that of Group B was 13.1 minutes with SD 1.64. Statistical analysis by students t-test showed a p value of < 0.05 which is statistically significant.

Mean onset time of sensory block in Group A was 4.53 minutes with SD of 1.25 and that of Group B was 8.5 minutes with SD 1.10. Statistical analysis by students t-test showed a p value of < 0.05 which is statistically significant.

Mean duration of sensory block in Group A was 874.67 minutes with SD of 28.61 and that of Group B was 632 minutes with SD 29.29. Statistical analysis by students t-test showed a $\mathrm{p}$ value of $<0.05$ which is statistically significant.
Mean duration of motor block in Group B was 528.67 minutes with SD of 29.33 and that of Group A was 807.67 minutes with SD 28.48. Statistical analysis by students t-test showed a $\mathrm{p}$ value of $<0.05$ which is statistically significant. Duration of analgesia is the time of injection of drugs to the first demand of analgesic. Duration of analgesia in Group A was 910.33 \pm 27.09 minutes while in group B it was $804.33 \pm 34.60$ minutes.

This shows a prolongation of analgesia by 96 minutes in the Group A compared to Group B.

Analysis by $t$ test shows that this difference in the duration of analgesia is highly significant with a $\mathrm{p}$ value of $<0.001$.

Figure 4 Graph showing Respiratory rate(RR) at various time intervals

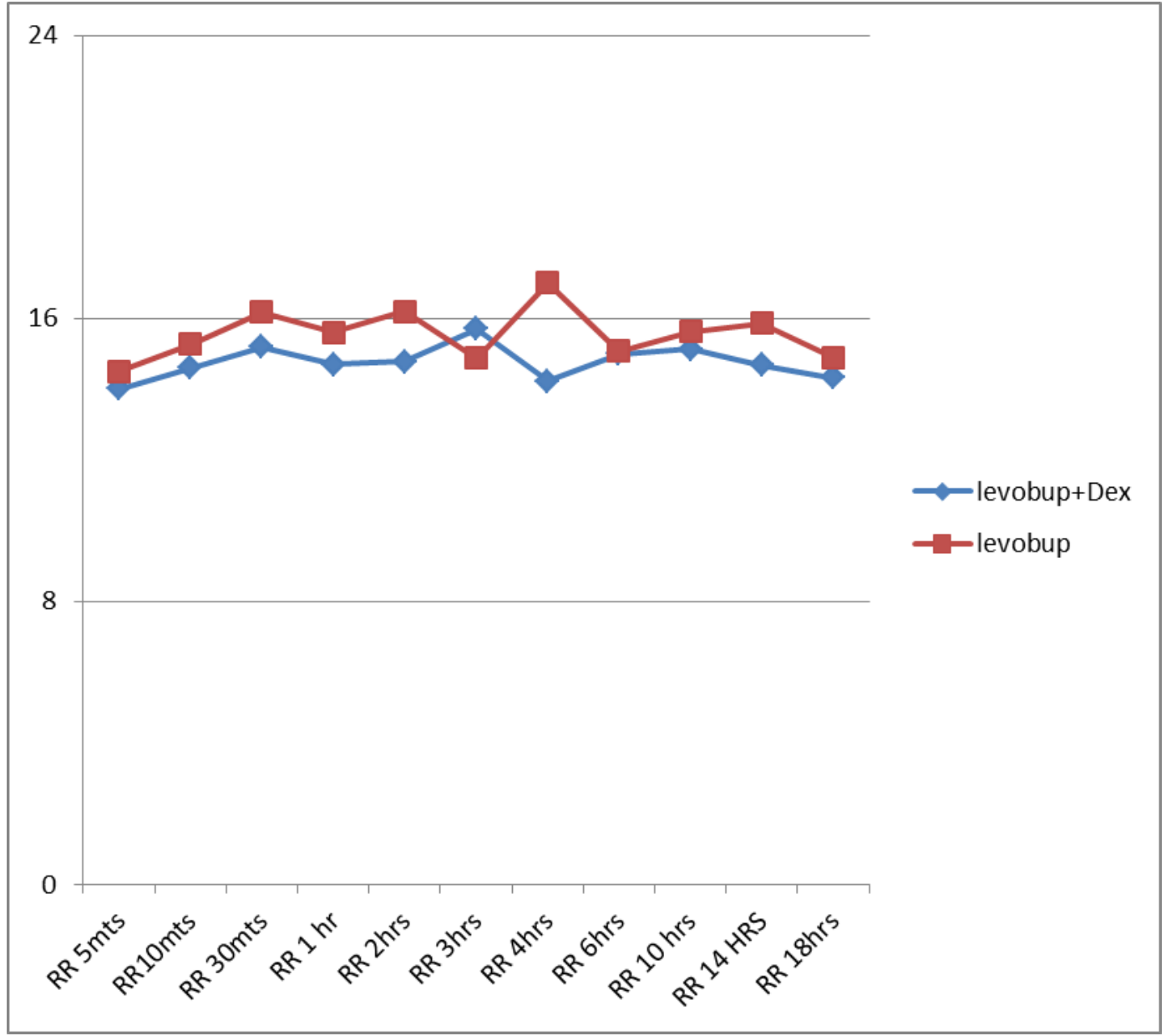

There is no statistically significant difference in respiratory rate between both the groups 
Figure 5 Graph showing oxygen saturation (SP2O) at various time intervals

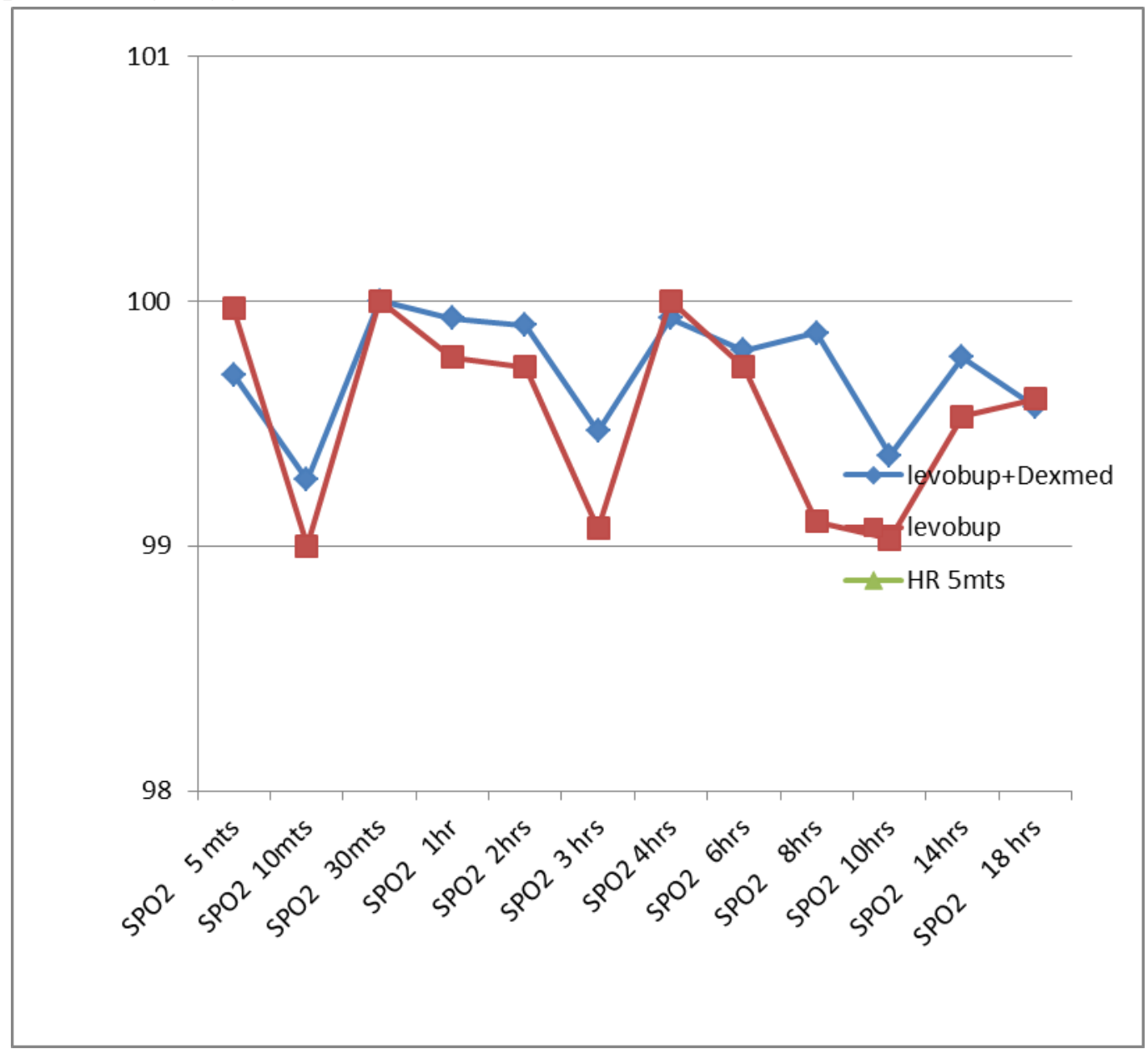

There is no statistically significant difference in oxygen saturation between both the groups

\begin{tabular}{|l|c|c|c|}
\hline & Group A & Group B & P-value \\
\hline Duration of analgesia & $910.33 \pm 27.09$ & $804.33 \pm 34.60$ & $<0.001$ \\
\hline Duration of sensory block & $874.67 \pm 28.61$ & $632 \pm 29.29$ & $<0.05$ \\
\hline Duration of motor block & $807.67 \pm 28.48$ & $528.67 \pm 29.33$ & $<0.05$ \\
\hline Onset of sensory block & $4.53 \pm 1.25$ & $8.5 \pm 1.10$ & $<0.05$ \\
\hline Onset of motor block & $8.67 \pm 1.68$ & $13.1 \pm 1.64$ & $<0.05$ \\
\hline
\end{tabular}

\section{DISCUSSION}

Levobupivacaine is a newer long acting amide local anesthetic found to be equally efficacious to bupivacaine, but with a better safety profile when used in brachial block. Dexmedetomidine has a unique mechanism of action it acts on alpha-2 adrenoceptors in the locus ceruleus ${ }^{12}$, Presynaptic activation of alpha-2Aadrenoceptors in the locus ceruleus inhibits the release of nor-epinephrine and results in the sedative and hypnotic effects ${ }^{13}$. Stimulation of alpha-2 adrenoceptors, an important modulator of nociceptive neurotransmission terminates the propagation of pain signals leading to analgesia. Postsynaptic activation of alpha-2 receptors in the CNS results in bradycardia ${ }^{11}$. Esmaoglu et al done a study on dexmedetomidine added to levobupivacaine prolongs axillary brachial plexus block .They conducted study on Sixty patients scheduled for elective forearm and hand surgery were divided into 2 equal groups in a randomized, double-blind fashion. The 4 main nerves in the axilla (musculocutaneus, radial, median, ulnar) were identified using neural stimulation. Patients were 
assigned randomly to 1 of the 2 groups. In group $\mathrm{L} \quad(\mathrm{n}=30), 40 \mathrm{~mL} \quad(200 \mathrm{mg})$ of $0.5 \%$ levobupivacaine $+1 \mathrm{~mL}$ saline and in group $\mathrm{LD}$ $(\mathrm{n}=30), \quad 40 \mathrm{~mL}(200 \mathrm{mg})$ of $0.5 \%$ levobupivacaine $+1 \mathrm{~mL}$ dexmedetomidine were given. Motor and sensory block onset times, block durations, and duration of analgesia were recorded. Sensory and motor block onset times were shorter in group LD than in group L. Sensory and motor blockade durations were longer in group LD than in group L. Duration of analgesia was longer in group LD than in group L. The study concluded that dexmedetomidine added to levobupivacaine for axillary brachial plexus block shortens the onset time and prolongs the duration of the block and the duration of postoperative analgesia ${ }^{14}$.Shivinder Singh and Amitabh Aggarwal done a randomized controlled double-blinded prospective study of the efficacy of clonidine added to bupivacaine as compared with bupivacaine alone used in supraclavicular brachial plexus block for upper limb surgeries. They also found an enhancement of perioperative analgesia and prolongation of recovery of sensation in the clonidine group, well beyond the pharmacological effect of either clonidine or bupivacaine significantly enhances the quality of supraclavicular brachial plexus block in upper limb surgeries by a faster onset and prolonged duration of sensory and motor block, enhancing post-operative analgesia. These benefits are not associated with any haemodynamic changes, sedation or other adverse effects ${ }^{15}$.

Foster et al conducted study on levobupivacaine: a review of its pharmacology and use as a local anaesthetic. In this study they concluded that Levobupivacaine is a long acting local anaesthetic with a clinical profile closely resembling that of bupivacaine. Excluding pharmaco economic considerations, levobupivacaine is an appropriate choice for use in place of bupivacaine ${ }^{16}$. Deepika Shukla et al conducted a study on intrathecal dexmedetomidine with intrathecal magnesium sulfate used as adjuvants to bupivacaine. A total of 90 patients classified as American Society of
Anesthesiologists status I and II scheduled for lower abdominal and lower limb procedures were prospectively studied. The onset time to reach peak sensory and motor level, the regression time for sensory and motor block, hemodynamic changes and side-effects were recorded. It was found that onset of anesthesia was rapid and of prolonged duration in the dexmedetomidine group (D). However, in the magnesium sulfate group (M), although onset of block was delayed, the duration was significantly prolonged as compared with the control group (C), but to a lesser degree than in the dexmedetomidine group (D). The groups were similar with respect to hemodynamic variables and there were no significant sideeffects in either of the groups ${ }^{17}$. Another study conducted by Saumya Biswas et al on Dexmedetomidine an adjuvant to levobupivacaine in supraclavicular brachial plexus Block: A randomized double blind prospective study on sixty patients scheduled for elective forearm and hand surgery were divided into two equal groups. The patients received brachial plexus block via supraclavicular route with the help of nerve stimulator. In group $\mathrm{L} \quad(\mathrm{n}=30) \quad 35 \mathrm{cc}$ of levobupivacaine with $1 \mathrm{ml}$ of isotonic saline and in group LD ( $\mathrm{n}=30$ ) 35cc of levobupivacaine with 1 $\mathrm{ml}$ of (100microgram) of dexmedetomidine was given. Results were Sensory and motor block durations were longer in group LD as compared to L. Duration of analgesia was significantly longer in group LD as compared to group L. They concluded that Dexmedetomidine added to levobupivacaine in supraclavicular brachial plexus block prolongs the duration of block and the duration of postoperative analgesia ${ }^{18}$.A study conducted by Chad $\mathrm{M}$. Brummett et al on Additives to Local Anesthetics for Peripheral Nerve Blockade .Many additives to local anesthetics to prolong the duration of analgesia for peripheral nerve blocks have been studied. In this review, the authors focus on the more commonly described additives, including epinephrine, clonidine, dexmedetomidine, buprenorphine, dexamethasone, tramadol, sodium bicarbonate, 
and midazolam ${ }^{19}$. While the primary focus of this review is the effect of the additive on the duration of analgesia, neurotoxicity and other safety concerns are also discussed.

\section{Patient characteristics across the groups}

The patients in our study groups did not vary much with respect to age. The type of surgeries performed was almost identical in both the groups. The study groups did not vary much with respect to duration of surgery.

\section{Changes in the perioperative cardiovascular parameters}

There were no significant differences between the study groups with respect to pattern of changes in heart rate, systolic blood pressure, diastolic blood pressure, mean arterial pressure perioperatively.

In the study conducted Kenan Kaygusuz et al done on Effects of Adding Dexmedetomidine to levobupivacaine in axillary brachial plexus block. They divided patients into two groups. Group L patients $(\mathrm{n}=32)$, an axillary block was performed with $39 \mathrm{~mL}$ levobupivacaine $5 \%$ plus $1 \mathrm{~mL}$ of isotonic sodium chloride. In group D patients $(\mathrm{n}=$ 32), an axillary block was performed with $39 \mathrm{~mL}$ levobupivacaine $5 \%$ and $1 \mathrm{~mL}$ dexmedetomidine $1 \mu \mathrm{g} / \mathrm{kg}-1$ plus isotonic sodium chloride, it was found that the hemodynamics remained stable in both the group L and group D for supraclavicular brachial plexus block ${ }^{22}$.

A study done by Damien B, Murhy et al reviews current evidence for the efficacy of adding novel analgesic adjuncts to brachial plexus block and the results were prolonged analgesic effect without the disadvantage of systemic side effects or prolonged motor block ${ }^{25}$.

we conclude that there is no difference between $0.5 \%$ Levobupivacaine + dexmedetomidine and $0.5 \%$ Levobupivacaine $+0.9 \%$ saline with respect to variation in hemodynamic parameters when used at equal volumes for supraclavicular brachial plexus block ${ }^{25}$.

\section{Onset time of Sensory and Motor block}

In our study, Mean onset time of sensory block in Group A was 4.53 minutes with SD of 1.25 and that of Group B was 8.5 minutes with SD 1.10.
Statistical analysis by student's-test showed a $\mathrm{p}$ value of $<0.05$ which is statistically significant. In our study, Mean onset time of motor block in Group A was 8.67 minutes with SD of 1.68 and that of Group B was 13.1 minutes with SD 1.64. Statistical analysis by students t-test showed a $\mathrm{p}$ value of $<0.05$ which is statistically significant. Sarita S Swami and colleagues conducted a randomised double-blind prospective study on Comparison of dexmedetomidine and clonidine as an adjuvant to local anaesthesia in supraclavicular brachial plexus block. The study concluded that dexmedetomidine when added to local anaesthetic in supraclavicular brachial plexus block enhanced the duration of sensory and motor block and also the duration of analgesia ${ }^{20}$.

Sandhya Agarwal and colleagues conducted a study on Dexmedetomidine prolongs the effect of bupivacaine in supraclavicular brachial plexus block. Study concluded that Dexmedetomidine added as an adjuvant to bupivacaine for supraclavicular brachial plexus block significantly shortens the onset time and prolongs the duration of sensory and motor blocks and duration of analgesia with no adverse effects ${ }^{22}$.

The above observations were similar to our study results. Hence, we conclude that Dexmedetomidine added as an adjuvant to Levobupivacaine for supraclavicular brachial plexus block significantly shortens the onset time of sensory and motor blocks.

\section{Duration of Sensory block and Motor block}

In our study Mean duration of sensory block in Group A was 874.67 minutes with SD of 28.61 and that of Group B was 632 minutes with SD 29.29. Statistical analysis by students t-test showed a $\mathrm{p}$ value of $<0.05$ which is statistically significant.Mean duration of motor block in Group B was 528.67 minutes with SD of 29.33 and that of Group A was 807.67 minutes with SD 28.48 . Statistical analysis by students t-test showed a $\mathrm{p}$ value of $<0.05$ which is statistically significant.Esmaoglu et al have done a study on Dexmedetomidine added to levobupivacaine prolongs axillary brachial plexus block, the study 
concluded that Dexmedetomidine added to levobupivacaine for axillary brachial plexus block shortens the onset time and prolongs the duration of the block and the duration of postoperative analgesia ${ }^{14}$.Saumya Biswas et al conducted study on Dexmedetomidine an adjuvant to Levobupivacaine in supraclavicular brachial plexus Block. They concluded that Dexmedetomidine added to levobupivacaine in supraclavicular brachial plexus block prolongs the duration of block and the duration of postoperative analgesia ${ }^{18}$.Piangatelli et al have done a study on Levobupivacaine and ropivacaine in the infraclavicular brachial plexus block. The study concludes that with levobupivacaine there was long duration of sensory block associated with the with good analgesia, less toxicity ${ }^{23}$.

The above observations were similar to our study results .Hence, we conclude that Dexmedetomidine added as an adjuvant to bupivacaine for supraclavicular brachial plexus block significantly shortens the onset time and prolongs the duration of sensory and motor blocks and duration of analgesia with no adverse effects.

\section{Duration of Analgesia}

Duration of analgesia is the time of injection of drugs to the first demand of analgesic. Duration of analgesia in Group B was $910.33 \pm 27.09$ minutes while in group B it was $804.33 \pm 34.60$ minutes.This shows a prolongation of analgesia by 96 minutes in the Group A compared to Group B.Analysis by $\mathrm{t}$ test shows that this difference in the duration of analgesia is highly significant with a $\mathrm{p}$ value of $<0.001$.Esmaoglu et al have done a study on Dexmedetomidine added to levobupivacaine prolongs axillary brachial plexus block, the study concluded that Dexmedetomidine added to levobupivacaine for axillary brachial plexus block shortens the onset time and prolongs the duration of the block and the duration of postoperative analgesia ${ }^{8}$. They concluded that that Dexmedetomidine added to levobupivacaine for axillary brachial plexus block prolongs the duration of analgesia.

\section{Adverse effects / complications}

No patient in our study developed any significant side effects. This signifies that adverse effects were not significant in both the groups. Agarwal S, Aggarwal R, Gupta done a study Dexmedetomidine prolongs the effect of bupivacaine in supraclavicular brachial plexus block. They concluded Dexmedetomidine added as an adjuvant to bupivacaine for supraclavicular brachial plexus block significantly shortens the onset time and prolongs the duration of analgesia with no adverse effects ${ }^{17}$.Mc Clellan, Spencer CM. conducted study on Levobupivacaine intraoperative hypotension was the most commonly reported adverse event with levobupivacaine and no serious arrhythmias, side effects occurred ${ }^{25}$.Anjan Das et al conducted study on different additives used to prolong brachial plexus block. The study concluded that adding dexmedetomidine to supraclavicular brachial plexus block increases the sensory and motor block duration and time to first analgesic use, and decreases total analgesic use with no sideeffects $^{26,27}$.

In above mentioned studies they got similar results as our study so we conclude that the side effects / complication like sedation ,hypotension, bradycardia were almost negligible with both group A and group B if right doses are used and properly deposited avoiding intravascular injection.

\section{BIBILIOGRAPHY}

1. Kulenkampff, D (1911). "Zur anästhesierung des plexus brachialis" [On anesthesia of brachial plexus]. 38: 1337-40.

2. Hirschel G (1911-07-18). "Die anästhesierung des plexus brachialis fuer die operationen an der oberen extremitat" [Anesthesia of the brachial plexus for operations on the upper extremity]. 58: 1555-6.

3. Kulenkampff, D; Persky, MA (1928). "Brachial plexus anaesthesia: its 
indications, technique, and dangers". Annals of Surgery 87 (6): 883-91

4. Winnie AP, Collins VJ. "The subclavian perivascular technique of brachial plexus anesthesia. Anesthesiology"1964;25:35-63

5. Burlacu CL, Buggy DJ. Update on local anesthetics: focus on levobupivacaine. Ther Clin Risk Manag. 2008 Apr;4(2): 381-92.

6. Kopacz DJ, Allen HW. Accidental intravenous levobupivacaine. Anesth Analg. 1999 Oct;89(4):1027-9.

7. Gristwood RW. Cardiac and CNS toxicity of levobupivacaine: strengths of evidence for advantage over bupivacaine. Drug Saf Int J Med Toxicol Drug Exp. 2002;25 (3):153-63.

8. Anju Grewal, Dexmedetomidine: new avenues. J Anaesthesiol Clin Pharmacol july - sept 2011;27: issue 3

9. Keira P. Mason Dexmedetomidine in Children: Current Knowledge and Future Applications Anesthesia-Analgesia 2011 .

10. Manpreeth kaur P M sing, Current role of dexmedetomidine in clinical anaesthesia and intensive care. Anaesthesia: Essays and Researches; 5 (2) July - Dec 2011

11. Jones MEP, Maze M. Can we characterize the central nervous system actions of alpha-2 adrenergic agonists? Br J Anaesth 2001;86(1):1-3.

12. Ebert TJ, Hall JE, Barney JA, Uhrich TD, Colinco MD. The effects of increasing plasma concentrations of dexmedetomidine in humans. Anaesthesiology 2009;93;382-94

13. Pavlin DJ , Rapp et al - Factors affecting discharge time in adult out patients. Anaesthesia Analgesia - 1998; 87:8161.

14. Esmaoglu A, Yegenoglu F, Akin A, Turk CY. Dexmedetomidine added to levobupivacaine prolongs axillary brachial plexus block. Anesth Analg. 2010 Dec;111(6):1548-51.
15. Singh S, Aggarwal A. A randomized controlled double-blinded prospective study of the efficacy of clonidine added to bupivacaine as compared with bupivacaine alone used in supraclavicular brachial plexus block for upper limb surgeries. Indian J Anaesth. 2010;54(6):552-7.

16. Foster RH, Markham A. Levobupivacaine: a review of its pharmacology and use as a local anaesthetic. Drugs. 2000 Mar;59(3): 551-79.

17. Shukla D, Verma A, Agarwal A, Pandey HD, Tyagi C. Comparative study of intrathecal dexmedetomidine with intrathecal magnesium sulfate used as adjuvants to bupivacaine. J Anaesthesiol Clin Pharmacol. 2011;27(4):495-9.

18. Biswas S, Das RK, Mukherjee G, Ghose T. Dexmedetomidine an Adjuvant to Levobupivacaine in Supraclavicular Brachial Plexus Block: A Randomized Double Blind Prospective Study. Ethiop J Health Sci. 2014 Jul;24(3):203-8.

19. Brummett CM, Williams BA. Additives to Local Anesthetics for Peripheral Nerve Blockade. Int Anesthesiol Clin. 2011;49(4):104-16.

20. Swami SS, Keniya VM, Ladi SD, Rao R. Comparison of dexmedetomidine and clonidine (?2 agonist drugs) as an adjuvant to local anaesthesia in supraclavicular brachial plexus block: A randomised double-blind prospective study. Indian $\mathbf{J}$ Anaesth. 2012;56(3):243-9.

21. Kaygusuz K, Kol IO, Duger C, Gursoy S, Ozturk H, Kayacan U, et al. Effects of Adding Dexmedetomidine to Levobupivacaine in Axillary Brachial Plexus Block. Curr Ther Res Clin Exp. 2012 Jun;73(3):103-11.

22. Agarwal S, Aggarwal R, Gupta P. Dexmedetomidine prolongs the effect of bupivacaine in supraclavicular brachial plexus block. J Anaesthesiol Clin Pharmacol. 2014 Jan;30(1):36-40. 
23. C P, C DA, L P, F R, P C, D T. Levobupivacaine and ropivacaine in the infraclavicular brachial plexus block. Minerva Anestesiol. 2006 Apr;72(4):21721.

24. Chan \{“id":5019604 V, first_name ": "Vincent, last_name“":"Chan, page_name“": "VincentChan, domain_name":"hkust, created_at":"’2013-08-01T20:20:58.402-

07:00, et al. Novel Analgesic Adjuncts for Brachial Plexus Block: A Systematic Review [Internet]. [cited 2014 Oct 17]. Available from: http://www.academia.edu/4155469/_Novel_ Analgesic_Adjuncts_for_Brachial_Plexus _Block_A_Systematic_Review

25. McClellan KJ, Spencer CM. Levobupivacaine. Drugs. 1998 Sep;56(3):355-62; discussion 363-4.

26. SaudiJAnaesh8572-8653997_022413.pdf [Internet]. [cited 2014 Nov 13]. Available from:

http://www.saudija.org/temp/SaudiJAnaes h8572-8653997_022413.pdf

27. SaudiJAnaesh62109-8747581_022547.pdf [Internet]. [cited 2014 Nov 13]. Available from:

http://www.saudija.org/temp/SaudiJAnaes h62109-8747581_022547.pdf 